Preprint

\title{
Transitioning to age inclusive early intervention for psychosis
}

\author{
O’Dris coll, Ciarán ${ }^{1}$ \\ Free, Katherine ${ }^{2}$ \\ Attard, Angelica ${ }^{3}$
}

\author{
Carter, Peter ${ }^{3}$ \\ Mas on, Jemma ${ }^{3}$ \\ Shaikh, Madiha ${ }^{1,3}$
}

\begin{abstract}
Abs tract
Aim: Early Intervention in psychosis Services (EIS) have previously restricted access based on age. However, there is now a move to age inclusive s ervice. Weaimed to examine differences between early and late onset (>35 years) psychosis to see if a threshold was valid. We also in vestigated the potential of a statistical modelling method to identify group characteristics which may be mis sed using a descriptive approach.

Method: Rou tine clinical data $(n=343)$, froman EIS, comprising sociodemo graphic, clinical, phy sical, and treatment variables, were examined using descriptive and classification and regres sion tree (CART) analys is .

Results: The findings suggest that age differences were best explained by s ocial factors. There was no emerging evidence that the differences exhibited had a fundamental impact on the clinical outc omes of the clients in terms of s upportbeyond EIS (i.e., hospitalisation and home treatment team involvement) and pharmacological and psychological in terventions. CART analysis revealed distinct service us er characteristics as sociated with the clinical outcomes.

Conclusion: There was no evidence to support a clinical cut off based on age providing support for age inclusive services. However, in the transition to age inclusive service delivery, EIS need to consider social/ life stage variables, adapting provision where service delivery may operate a youth focus ed model. Routine analysis of clinical data should employ methods to identify groups of service users who may require adjusted service provision..
\end{abstract}

Supplementary material: https://osf.io/m6wpe/

Keywords: Early Intervention, psychosis, late onset

Early intervention services (EIS) were nationally implemented in England in the early 2000s (Cheng et al., 2011; Radhakrishnan et al., 2018) to improve access to treatment for those who experience a first episode of psychosis (FEP) FEP occurs most commonly in late adolescence and early adulthood, and as a result the services were initially commissioned for individual's aged 14 to 35 years old (Kirkbride et al., 2006). EIS offers better management of FEP than standard treatment (Nordentoft et al., 2014) and are cost effective (Radhakrishnan et al., 2018). However, the evidence is restricted to those within the age bracket.

More recent evidence revealed that the clinical and functional needs of older individuals with FEP were just as great, thus demonstrating a need to expand the

Affiliations

1 Research Department of Clinical, Educational and Health Psychology, University College London, London, UK

2 Division of Psychiatry, University College London, London, UK

3 North East London NHS Foundation Trust, London, UK age access to EIS (Lappin et al., 2016; National Institute for Clinical Excellence, 2016). As a result, there has been a shift to age inclusive (14 to 65) EIS in the UK. Older service users $(>35)$ with FEP are an understudied group in comparison to the younger cohort. Older FEP individuals have distinguishing features and characteristics (Clay et al., 2018), and thus are likely to have different needs and expectations in terms of treatment and support compared to the traditional EIS client population, such as different aims in psychological therapy or vocational objectives (Golay et al., 2017; Greenfield et al., 2018; Selvendra, Baetens, Trauer, Petrakis, \& Castle, 2014). A recent study (Jagger et al., 2019) looking at treatment components utilization in an age inclusive service observed more complex and sustained needs in service users over the age of 35 , especially in terms of care coordination. As a result, it has been suggested that the benefits of EIS cannot necessarily be generalised or extrapolated to older individuals (Mitford, Reay, McCabe, Paxton, \& Turkington, 2010) and monitoring the change and expansion of service user focus in EIS 
is important. Age inclusivity will inevitably result in recommissioning of EIS services to better accommodate the complex clinical and social needs of the service users.

\section{Objectives}

We aim to assess differences in sociodemographic, clinical, and physical characteristics between the under and 35 FEP groups currently using services, evaluating differences and similarities in clinical or functional needs of service users and service provision (specifically pharmacological and psychological interventions).

\section{Methodology}

The study was approved in North East London Foundation Trust under audit and service evaluation: it informed the further development of EIS service delivery.

Anonymised data were collected for all service users $(n=343)$ with a FEP within four boroughs of North East London Foundation Trust. The boroughs began the transition to an ageless service in 2018. Service users over 35 years of age, had previously resided within recovery teams but could access psychological therapies through the EIP team. Data were extracted on a wide range of physical, clinical, and sociodemographic characteristics through the electronic patient record system RiO and manual extraction.

Sociodemographic characteristics included gender, age, nationality, ethnicity, marital status, religion, first language, child dependants, and employment. Clinical characteristics included diagnosis, source of referral, and hospital admissions, hospital length of stay, substance use, medication type and dose, psychological therapy uptake and engagement. Lastly physical characteristics included, weight, height, BMI, blood glucose, cholesterol, blood pressure, smoking status, alcohol use, and substance use.

Psychological interventions included the provision of Cognitive Behavioural Therapy for Psychosis (CBTp), Family Intervention for Psychosis (FIp), and Carer Well-being Intervention (CWI).

Routine clinical data is often, complex, unbalanced, and containing missing values and this poses challenges to exploring the impact of service expansion. Relationships between variables may not be linear and may involve high-order interactions. Decision trees (CART) may be able to manage these challenges and are suited for both exploring and modelling routine clinical data. Decision trees are relatively simple to interpret and elucidate clear and meaningful groups within data.

\section{Analysis}

Between group analyses compared service users above and below 35 years across all variables. CARTs were conducted in R, using rpart (Therneau, Atkinson, $\&$ Ripley, 2018). Three different models were run for illustrative and explanatory purposes, utilising count, continuous, and binary explanatory variables.

A classification tree was used to investigate the relationship between variables and engagement with psychological therapy (binary) where the node represents the predicted class. Regression trees were used for antipsychotic dosage (continuous) and age (continuous) where the node represents the mean response, and a poisson tree for intensive support (count: sum of admissions to hospital and home treatment team referrals) characterized by the estimated response rate.

The 'tree' is constructed by repeatedly splitting the data, stepwise, starting with the best single variable into two groups, and this process applied to the subgroups, until they reach a minimum size. The objective is to partition the response into homogeneous groups, but also to keep the tree reasonably small. This tree can be grown until every person is classified, where the size of a tree equals the number of final groups. The tree displays the splits, and in each node it displays the response (e.g. event response) and the percentage of the sample.

This model runs the risk of overfitting; modelling idiosyncrasies or noise in the data (so some splits may be meaningless). Running analysis to reduce overfitting results in a shallower tree (pruning) to reach the 'optimal' depth. Pruning was conducted using tuning parameters and 10 fold cross-validation error rate estimation (complexity parameter), where a split which does not improve the model fit is not pursued. The minimum number of observations in each node before a split is attempted was set to 20 with the minimum number of observations allowed in a node set to 5 . As the purpose was to ensure real world applicability of the modelling, where missing data existed, no imputation or surrogate methods were used. Instead, where a service user is missing the primary split variable, they do not progress further down the tree.

\section{Results}

Complete descriptive tables are included in supplementary material. 


\section{Sociodemographic characteristics}

Of these service users $258(75.2 \%)$ were in the under 35 group and $85(24.8 \%)$ were classified as over 35 . Genders were well represented; $53.4 \%$ of the sample was male. The mean age for the under 35 group was 24.88 (5.33) and for the over 35 was 45.13 (7.58) years. The most common ethnicities in the sample were White (31.2\%), Black African/ Caribbean/ British (29.7\%), and Asian/Asian British (22.4\%).

There were significant sociodemographic differences between the age groups. Under 35s were more likely to be male $(\mathrm{p}<.001)$. Over 35 s were more likely to have child dependants under the age of $18(\mathrm{p}=.043)$ and live independently $(\mathrm{p}<.001)$. A significant difference $w$ as found for marital status $(\mathrm{p}<.001)$. Under $35 \mathrm{~s}$ were more likely to be single and less likely to be married or cohabiting with a partner. There were significant differences in employment status among the two age groups $(p<.001)$. Being a student was more common amongst under $35 \mathrm{~s}$ and unemployment was higher amongst over $35 \mathrm{~s}$.

\section{Physical Characteristics}

Cholesterol levels were higher in over $35 \mathrm{~s}$ $(\mathrm{p}=.003)$. They were more likely to have a cholesterol level of over five $\mathrm{mmol} / \mathrm{L}$; above UK government recommendations $(\mathrm{p}=.009)$. Furthermore, logistic regressions revealed a significant difference in frequency of elevated blood pressure between the under and over $35 \mathrm{~s}$ ( $4.7 \%$ v. $11.8 \%$ respectively), high blood pressure was more common in the over $35 \mathrm{~s}(\mathrm{p}=.01)$. Elevated blood pressure was recorded if blood pressure was higher than $140 / 90 \mathrm{mmHg}$. The analysis also revealed that general substance misuse was more common in under $35 \mathrm{~s}(36.8 \%$ v. $15.3 \%$, p<.001), specifically cannabis $(30.2 \%$ v. $9.4 \%, \mathrm{p}<.001)$. Significant differences in mean BMI and weight were not demonstrated between age groups. The analyses did not reveal significant differences for alcohol misuse, smoking, diabetes or blood glucose.

\section{Clinical Characteristics}

\section{Diagnosis}

Delusional disorder was more frequently diagnosed in the older cohort (1.2\% for under $35 \mathrm{~s} \mathrm{v} .8 .2 \%$ for over $35 \mathrm{~s}$ ), whereas drug induced psychotic disorder was more common in the under $35 \mathrm{~s}(5.8 \% \mathrm{v}$. $0 \%$ for over 35s). Moreover, under 35s were more likely to have a diagnosis of unspecified nonorganic psychosis $(30.2 \%$ v. $9.4 \%)$. Differences in affective disorder diagnosis were also significant $(\square 2(6)=14.513$, $\mathrm{p}=.024)$. Over $35 \mathrm{~s}$ were more likely to have a diagnosis of severe depressive episode with psychotic symptoms $(14.1 \%$ v. $4.3 \%)$.

\section{Hospital admission}

Under $35 \mathrm{~s}$ were more likely to stay in hospital for longer following an admission. This was found for both average hospital stays (22.9 days for under $35 \mathrm{~s} \mathrm{v}$. 9.9 days, $\mathrm{p}<.001$ ), and total stay (28.6 days for under $35 \mathrm{~s}$ v. 12.8 days, $\mathrm{p}<.001)$. However, no significant differences were found between groups for actual or mean number of hospitalisations.

Medication

There were no significant variations between age groups for antipsychotic prescription or chlorpromazine dose equivalents (mg). Antidepressant medication was more common in the over $35 \mathrm{~s}(\mathrm{p}=.014)$ and they were more likely to have higher fluoxetine dose equivalents $(\mathrm{mg})(15.19 \mathrm{mg}$ v. $7.81 \mathrm{mg} ; \mathrm{p}=.01)$.

Psychology

FIp uptake was $15.9 \%$ for under 35 s and $10.6 \%$ for over $35 \mathrm{~s}$. There were no significant differences between groups for FIp uptake, engagement or number of sessions attended.

CBTp uptake was similar with $64 \%$ of people under 35 accepting CBTp and $55.3 \%$ of people over 35 accepting CBTp. Under 35s were more likely to continue to attend and engage in CBTp (45.7\% v. $27.1 \%$; $\mathrm{p}=.003$ ).

Carers of under $35 \mathrm{~s}$ were more likely to accept the CWI for carers of individuals with psychosis compared to $27.1 \%$ of carers of over $35 \mathrm{~s} \mathrm{(39.9 \%} \mathrm{v.} 27.1 \%$; $\mathrm{p}=.034)$. There were no differences between groups in engagement or number of sessions attended.

\section{CART analyses \\ Age}

To investigate characteristics related to age, a regression tree (Figure 1.), where nodes represent the mean age and percentage of sample, was generated. The primary split for age, was marital status, and age differences reflected factors associated with life stage rather than illness or engagement factors. 


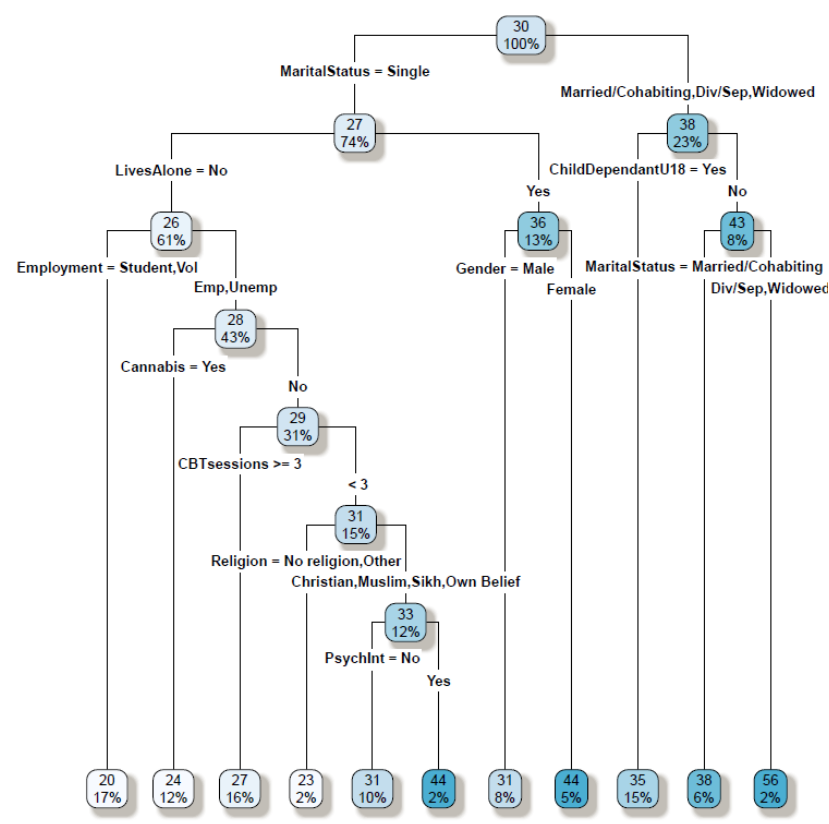

Figure 1. Pruned regression tree for age. Each node contains the mean age and percentage of the sample.

\section{Psychological intervention}

A binary classification tree (Figure 2.) indicates whether the service user or a family member engages (attending 2 or more sessions) in a psychological intervention (CBTp, FIp, or CWI). In this tree, there was a 50\% probability of engagement in psychology. Age was the primary split, and at a cut off of 55 years, $92 \%$ of those over 55 were engaged with psychology but this was a small group $(n=11)$. The largest group (18\%) who did not engage with psychology were those who were under 55, had English as a first language, BMI>20, had not been admitted to hospital at first episode and had less than 2 subsequent admissions, had no substance misuse problems and were in main stream housing (there was only a $23 \%$ probability that they would engage). Ethnicity was also indicated in this branching but was less informative as the split separated 'other'.

The greatest probability for engagement (92\%) was for those under 55, who did not have English as a first language, were employed/unemployed and ethnic ally black, mixed or other. While the least likely to engage were women under 30, who did not have English as a first language, were employed/unemployed and ethnically white or Asian (3\% of the sample, probability of $11 \%)$.
For antipsychotic treatment dosage, there was a high percentage of missing data. While more extensive trees could be generated, the tree with the best complexity parameter fit was based solely on whether the service user had been involved with the Home Treatment Team $(n=155: 64 \%$ yes, mean antipsychotic dosage $179 \mathrm{mg} ; 36 \%$ no, mean antipsychotic dosage $=113 \mathrm{mg}$ ).

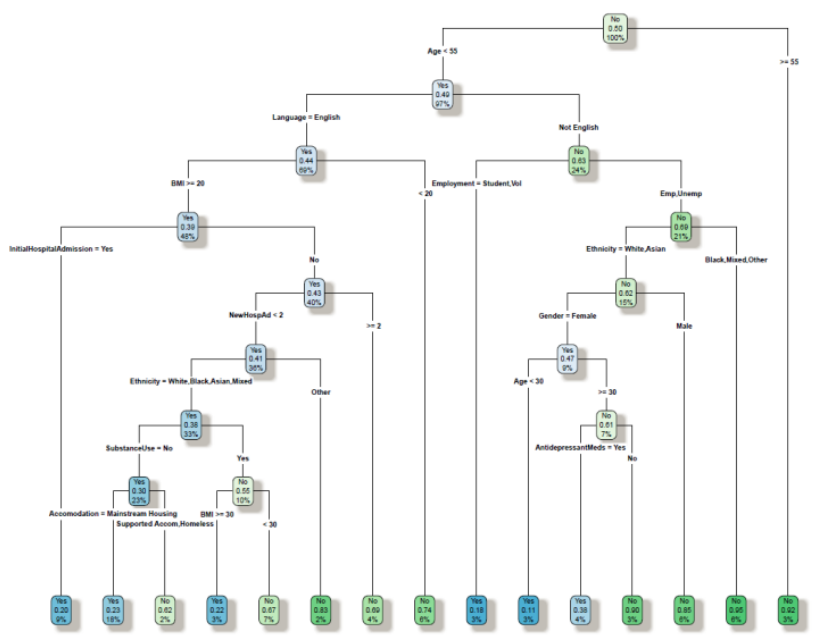

Figure 2. Pruned classification tree for engagement in a psychological intervention. Each node contains the predicted probability of engagement in psychological intervention and the percentage of the sample.

\section{Intensive support}

A composite variable including number of hospital admissions following first episode and home treatment team referrals was used to assess factors relating to intensity of support. A poisson regression tree (Figure 3) was estimated, where node represents the number of events and percentage of sample. Unremarkably, the primary split was antipsychotic dosage. This tree highlights that those requiring most intensive support (6 events) are in supported accommodation. For those in mainstream housing or homeless, factors associated with greater intensive support were smoking, being over 21, and a BMI >27 (4.5 events).

\section{Antipsychotic dosage}




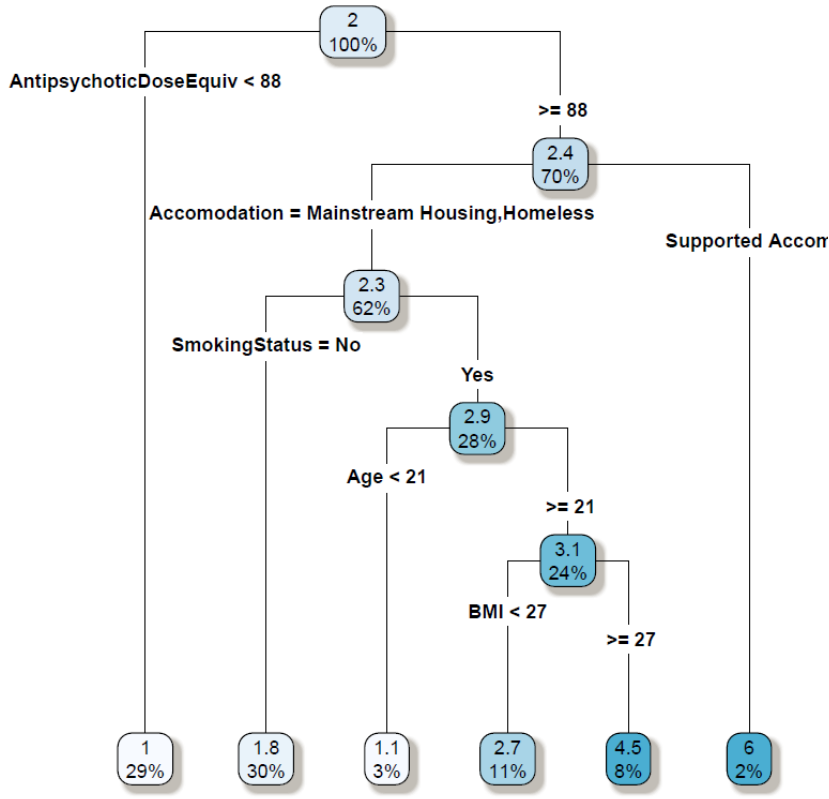

Figure 3. Pruned poisson tree for intensive support (number of hospitalisations and home treatment team involvement). Each node contains the expected number of events and the percentage of the sample.

\section{Discussion}

The present study is the first to explore differences in sociodemographic, physical health, and clinical characteristic setween individuals with early and late onset FEP utilizing a multivariate approach.

The findings suggest that while age differences were best explained by social factors, there were some interesting differences between the age groups in relation to diagnosis, employment rates, substance use, CBTp engagement, CWI uptake, and hospital length of stay. However, there was no emerging evidence that the differences exhibited had a fundamental impact on the clinical outcomes of the clients in terms of pharmacological and psychological interventions, and support beyond EIS (i.e. hospitalisation and home treatment teams).

Age was identified within the regression tree as a primary split for engaging with psychology, elucidated that the small proportion of those over 55 (3\% of the total sample) had a high probability in engaging with a psychological therapy. Interestingly, smaller and potentially overlooked subgroups were less likely to engage such as Asian and White women under 30, who did not have English as a first language, and were not in education or volunteering. It was also notable that along the branch for those women over 30, the use of antidepressant medication was associated with only $38 \%$ probability of engaging with psychology compared with $90 \%$ of those not taking antidepressant medication.

Those in supported accommodation had more hospitalisations and home treatment team referrals. Given the cross-sectional nature of the analyses it was difficult to discern whether this reflects a need being provided (i.e. the supported accommodation) or extra support being required for these individuals. It is also unclear how informative the next branch of the intensive support tree was, where those who are in mainstream housing or homeless, smoking, over $21 \mathrm{with}$ a BMI>27, had 4.5 events compared to those with BMI $<27$ having 2.7 events. Either BMI for those over 27 (more than $20 \%$ overweight) represents moderate health risks or may be a proxy for another variable not represented within the dataset.

The study provides support to the existing evidence (Greenfield et al., 2018; Selvendra et al., 2014) that the focus should be factors other than age. Age inclusivity introduces a greater complexity to the needs of services users, for example age represents a proxy for clear social needs, where those who are older are more likely to have child dependents, to be unemployed, and in a relationship. The increased number of child dependents for older clients may mean that services will be working with a larger number of young carers. It is important that EIS teams are aware of the right support services that exist for these individuals and are encouraging these carers to utilise them. It is important for EIS to be able to support the needs and accessibility of services by examining the impact of introducing age inclusivity in EIS, not only by using age as a proxy but also exploring sociodemographic, clinical and social characteristics that have important implications on clinical outcomes.

Our findings highlight the need to focus on vocational support and to offer different approaches based on differences in employment. Under 35s were more likely to be students and thus vocational support might relate to school, university or establishing a career, whereas support for the over $35 \mathrm{~s}$ might entail support for returning to work (Greenfield et al., 2018). The presence of higher unemployment rates in older individuals, also emphasises the importance of vocational support in this older cohort. This is supported by research that highlights the importance of employment for late onset FEP and discovered that financial needs 
and returning to work are critical in their recovery (Woodside \& Krupa, 2010). Although NICE advocates employment support as part of the EIS model, a national audit (Royal College of Psychiatrists, 2018) reported that only $46 \%$ of those unemployed and actively seeking work, were offered appropriate employment support.

While those over 35 had access to similar care as the under $35 \mathrm{~s}$, diagnostic differences may reflect pathways into care where, those over 35 would have been diagnosed within the community mental health teams and those under 35 in EIS. The epistemological approach to diagnosis is different between these teams.

While physical characteristics were not greatly different between the two age groups, these results cannot be reliably interpreted given the level of missing data. However, over $35 \mathrm{~s}$ did display elevated cholesterol levels and blood pressure, in line with international literature indicating greater likelihood of metabolic difficulties (Selvendra et al., 2014). Improvements in data collection and reporting are needed to help optimize service user's standard of care, to ensure all their physical health needs are met, and to understand whether physical health data are missing because physical health checks are not being carried out to the expected level, or data are not being well recorded. An additional consideration relates to how prepared services are to meet these additional physical health needs, especially where the youth focused model predominates.

Uptake of CBTp and FIp (attending 1 or more sessions) was relatively high for both groups although differences in CBTp engagement were observed. Overall FIp uptake remains low across age groups and this is not dissimilar to uptake nationally (Royal College of Psychiatrists, 2018). It is important to continue to address the nationally low uptake of FIp for both client groups as this intervention has important implications for decreasing the risk of relapse rates. Equally there is a need to clearly establish a definition of uptake to compare across studies.

Carers of under $35 \mathrm{~s}$ were more likely to accept CWI. How ever, there were no differences in engagement for those who did accept CWI, suggesting that once a carer had started an intervention, they were equally likely to remain engaged. EIS should continue to actively encourage carers of over $35 \mathrm{~s}$ to accept
CWI. It would be of value to understand if the lower uptake of CWI in the older cohort is due to their rejection of an offer or not being offered the intervention. This could highlight potential inequity in EIS and suggest that a change in approach is needed, or that obstacles to uptake of CWI need to be addressed.

\section{Limitations and future considerations}

The sample is composed of a diverse sociodemographic population across four boroughs in greater London and may not be generalisable. Nonetheless our findings are similar to those reported by Selvendra et al., (2014) and Greenfield et al. (2018), and highlight distinct social needs in the older cohort. The methods also offer an approach to capitalise on extracting meaningful data from routine clinical audits. The dataset reflects the reality of routinely collected clinical data, however, missingness precludes a comprehensive assessment of all the factors. CART analysis manages missing data more appropriately than the between groups analyses, however, it is likely that missingness was non-random. The data did not include assessment of duration of untreated psychosis, domains of psychosis, symptom severity, and trauma exposure which have been shown to be important variables in other studies (Greenfield et al., 2018; Üçok \& Bikmaz, 2007). The cross-sectional design does not allow for tracking predictors of relapse and remission which would be an important consideration when considering age of onset.

It $w$ as possible to identify more discrete clusters of service users within the data. The resulting models represent the systematic structure of the data as simply as possible. This allows for identifying hidden or neglected groups with the potential (when used in clinical practice) to identify individuals and address discrepancies in care provision and provide a more personalised approach to care. CART reduces overfitting and type 1 error providing a parsimonious approach to describing and exploring data. How ever, the depth to which a tree is interpreted, and variables included may reduce direct clinical relevance and despite care taken with model parameters, the model is limited by the lack of cross-validation in an independent sample.

\section{Conclusion}

Overall, age may be an arbitrary factor on which to determine and influence EIS delivery. While certain characteristics associated with age need to be considered, a primary focus on age (especially the $>35$ distinction) negates a more nuanced assessment of ser- 
vice user characteristics which are more likely to determine engagement and support need.

\section{Ack nowledgements}

We wish to thank Shanley Lew is and Tiffany Jade Sachak for their assistance in extracting the data.

\section{Conflict of interest statement}

The authors declare that they have no competing interest.

\section{References}

Cheng, F., Kirkbride, J. B., Lennox, B. R., Perez, J., Masson, K., Lawrence, K., ... Jones, P. B. (2011). Administrative incidence of psychosis assessed in an early intervention service in England: first epidemiological evidence from a diverse, rural and urban setting. Psychological Medicine, 41(05), 949-958. https://doi.org/10.1017/S0033291710002461

Clay, F., Allan, S., Lai, S., Laverty, S., Jagger, G., Treise, C., \& Perez, J. (2018). The over-35s: early intervention in psychosis services entering uncharted territory. BJPsych bulletin, 42(4), 137-140. https://doi.org/10.1192/bjb.2018.28

Golay, P., Alameda, L., Mebdouhi, N., Baumann, P., Ferrari, C., Solida, A., ... Conus, P. (2017). Age at the time of onset of psychosis: A marker of specific needs rather than a determinant of outcome? European Psychiatry, 45, 20-26. https://doi.org/10.1016/j.eurpsy.2017.06.002

Greenfield, P., Joshi, S., Christian, S., Lekkos, P., Gregorowicz, A., Fisher, H. L., \& Johnson, S. (2018). First episode psychosis in the over $35 \mathrm{~s}$ : is there a role for early intervention? Early Intervention in Psychiatry, 12(3), 348-354. https://doi.org/10.1111/eip.12322

Jagger, G., de la Fuente-Tomas, L., Stoch1, J., Allan, S. M., Clay, F., Kenedler, L., ... \& Perez, J. (2019). Early Intervention in Psychosis Treatment Components Utilization in Patients Aged Over 35. Community Mental Health Journal, 1-5.

Kirkbride, J. B., Fearon, P., Morgan, C., Dazzan, P., Morgan, K., Tarrant, J., ... Jones, P. B. (2006). Heterogeneity in Incidence Rates of Schizophrenia and Other Psychotic Syndromes. Archives of General Psychiatry, 63(3), 250. https://doi.org/10.1001/archpsyc.63.3.250

Lappin, J. M., Heslin, M., Jones, P. B., Doody, G. A., Reininghaus, U. A., Demjaha, A., ... Morgan, C. (2016). Outcomes following first-episode psychosis - Why we should intervene early in all ages, not only in youth. Australian and New Zealand Journal of Psychiatry, 50(11), 1055-1063. https://doi.org/10.1177/0004867416673454
Mitford, E., Reay, R., McCabe, K., Paxton, R., \& Turkington, D. (2010). Ageism in first episode psychosis. International Journal of Geriatric Psychiatry, 25(11), 1112-1118. https://doi.org/10.1002/gps.2437

National Institute for Clinical Excellence. (2016). Implementing the early intervention in psychosis access and waiting time standard: Guidance.

Nordentoft, M., Rasmussen, J. Ø., Melau, M., Hjorthøj, C. R., \& Thorup, A. A. (2014). How successful are first episode programs? A review of the evidence for specialized assertive early intervention. Current opinion in psychiatry, 27(3), 167-172.

Radhakrishnan, M., McCrone, P., Lafortune, L., Everard, L., Fowler, D., Amos, T., ... Birchwood, M. (2018). Costeffectiveness of early intervention services for psychosis and fidelity to national policy implementation guidance. Early Intervention in Psychiatry, 12(4), 747-756. https://doi.org/10.1111/eip.12481

Royal College of Psychiatrists. (2018). National Clinical Audit of Psychosis - National Report for the Core Audit. London.

Selvendra, A., Baetens, D., Trauer, T., Petrakis, M., \& Castle, D. (2014). First episode psychosis in an adult area mental health service-A closer look at early and late-Onset first episode psychosis. Australasian Psychiatry, 22(3), 235-241. https://doi.org/10.1177/1039856214532558

Therneau, T., Atkinson, B., \& Ripley, B. (2018). rpart: Recursive Partitioning and Regression Trees. R package.

Üçok, A., \& Bıkmaz, S. (2007). The effects of childhood trauma in patients with first-episode schizophrenia. Acta Psychiatrica Scandinavica, 116(5), 371-377.https://doi.org/10.1111/j.16000447.2007.01079.x

Woodside, H., \& Krupa, T. (2010). Work and financial stability in late-onset first-episode psychosis. Early Intervention in Psychiatry, 4(4), 314-318. https://doi.org/10.1111/j.1751 7893.2010.00189.x 